\title{
Model for the prediction of 3D surface topography in 5-axis milling
}

Sylvain Lavernhe

LURPA - ENS de Cachan - Université Paris Sud 11

61 avenue du Président Wilson

94235 Cachan cedex - France

tel: +33147402985

fax: +33147402220

lavernhe@lurpa.ens-cachan.fr

Yann Quinsat

LURPA - ENS de Cachan - Université Paris Sud 11

61 avenue du Président Wilson

94235 Cachan cedex - France

tel: +33147402213

fax: +331474022 20

quinsat@lurpa.ens-cachan.fr

Claire Lartigue

LURPA - ENS de Cachan - Université Paris Sud 11

61 avenue du Président Wilson

94235 Cachan cedex - France

IUT de Cachan - Université Paris Sud 11

9 avenue de la Division Leclerc

94234 Cachan cedex - France

tel: +33147402986

fax: +33147402220

lartigue@lurpa.ens-cachan.fr

Abstract:

The paper deals with the prediction of the 3D surface topography obtained in 5-axis milling in function of the machining conditions. For this purpose, a simulation model for the prediction of machined surface patterns is developed based on the well-known N-buffer method. As in sculptured surface machining the feed rates locally vary, the proposed model can be coupled to a feed rate prediction model. Thanks to the simulation model of 3D surface topography, the influence of the machining strategy on resulting 3D surface patterns is analyzed through an experimental design. Results enhance the major influence of the tool inclination on 3D topography. Surface parameters used in the study are strongly affected by the variation of the yaw angle. The effect of the feed rate is also significant on amplitude parameters. Finally, the analysis brings out the interest of using surface parameters to characterize 3D surface topography obtained in 5-axis milling.

Keywords:

Surface topography, surface patterns, 5 axis machining, machining strategy 


\section{Introduction}

Within the field of aeronautics, mold and die, or automotive industries, 3D sculptured surfaces are now widely used for the design of complex workpieces. To reach high surface quality, sculptured surfaces are generally obtained using a High-Speed Machining (HSM) process. Due to the shape complexity, multi-axis milling is more often used. Moreover, the tool trajectory is generated via CAM software which offers various machining strategies depending on the geometry of the surface to be machined. The machined surface quality thus results from the choice of the strategy and the corresponding cutting parameters.

As surface quality is concerned, some works have emphasized the importance of 3D surface topography, in particular in engineering applications [1]. 3D surface topography influences mechanical and physical properties of contacting parts, and plays a major role in surface integrity thus affecting fatigue life. Surface topography characterization highlights two major issues of same importance: the definition of relevant parameters characterizing 3D surface patterns resulting from multi-axis HSM, and the evaluation of the relationships linking the machining strategy (cutting conditions, machining direction and tool inclination) with the surface topography.

Concerning the first issue, the definition of parameters accounting for the 3D nature of the surface roughness has become necessary. A standardized project [ISO 25178-2:2007] proposes such parameters as a 3D extension of the classical 2D parameters, with a similar definition of those defined by the standard [ISO 12085:1996]. Among all, 12 parameters are more often used, which have been classified into 4 main families: amplitude parameters, spatial parameters, functional parameters and hybrid parameters (table 1). Some works focus on the characterization of the surface geometry using fractal dimensions [2] [3]. Fractal dimension is a well known indicator of the surface complexity: the fractal dimension increases following the surface roughness. Other authors propose models to describe surface topography by introducing conformal equivalent structure [4][5], this notion is introduced by comparing the slope or gradient distributions of surface topography.

Tab. 1 3D surface parameters

\begin{tabular}{|c|c|}
\hline & Surface parameters \\
\hline Amplitude parameters & Sq, Sz, Ssk, Sku \\
\hline Functional parameters & Sci, Svi, Sbi \\
\hline Spatial parameters & Sal, Std, Str, Sds \\
\hline Hybrid parameters & S $\Delta \mathrm{q}$ \\
\hline
\end{tabular}

However, a few studies try to link the surface roughness with the surface functionality through the 3D surface roughness parameters. For friction in servo hydraulic assemblies, surfaces with negative skewness, low kurtosis values, and high valley fluid retention index were found to have lower frictional characteristics [6]. The functionality of automotive cylinder bores is partially characterized by oil consumption and blow-by. For this application, Sq, Sk, Svk, $\mathrm{Sds}$, Sbi are more significant to describe oil consumption whereas Sv, Svi are well-adapted for blow-by [7]. Concerning the fatigue limit, it seems more interesting to consider Sq, Std and $\mathrm{Sal}$ [8]. The use of 3D parameters to 
characterize 3D surface topography is now efficient. Nevertheless, due to the lack of known information binding parameters to the function of the surface, the description of the 3D pattern obtained after machining remains essential.

Few formalized studies exist on the evaluation of the relationships linking machining strategy with surface topography [9]. Studies are generally performed considering an experimental standpoint or a simulation standpoint.

Concerning the experimental standpoint most results are qualitative, aiming at linking the surface quality with machining parameters. For instance, Ramos et al. [10] present observations concerning the influence of the machining direction on the surface quality after the machining of a boat propeller. In [11] authors present various topographies obtained on surfaces machined using different tool orientations. After the analysis of pattern 2D pictures, authors suggest avoiding upward and downward milling. Indeed, machining in the slope direction involves more marks on the part. Baptista and Antune Simoes [12] determine a model linking the feed rate, the transversal step and the surface roughness $\mathrm{Ra}=\mathrm{F}(\mathrm{fz}, \mathrm{p})$. The model obtained from experiments is only adapted for ball-end machining of aluminium alloy parts. Other works extend the model by adding the influence of the part geometry, such as the surface orientation [13] [14]. Results obtained show a variation of the surface quality according to the feed rate and the orientation. But these variations are not significant compare with measurement errors [15]. To summarize, experimental studies lead to results for which the field of validity is strongly linked with experimental conditions. None of them propose to clearly link the machining strategy with the surface topography.

In comparison, theoretical models offer a larger field of validity. Most models attempt to predict the surface roughness in function of the cutting conditions. The objective is to find the best cutting conditions leading to the required surface quality. Kim and Chu [16] describe the texture obtained in milling using the texture superposition model. A generalized cutter model is adopted introducing the effect of the fillet radius. In this approach, authors investigate the influence of the feed rate, the cutter type and the runout effect. As a conclusion, authors highlight the interest of using a filleted end-mill to reach better surface quality when the path interval is under a critical value. Bouzakis et al. take the influence of the tool orientation into account and focus on the motion of the cutting edge. The cutting flute is segmented into elementary linear sections of constant width. Simulations show the influence of the tool orientation, the transversal step and the feed rate on the surface quality [17]. Toh supplements this work by defining the best direction to machine an inclined plane [18]. The prediction model proposed by Chen et al. relies on the mathematical description of the cutting edge locus during ball-end machining [19]. Authors focus on the influence of the feed/pick ratio, the tool inclination and the initial cutting edge entrance angle. They highlight the importance of the feed interval in HSM. Moreover an optimisation of the feed rate is proposed according to the tool inclination. Liu et al. detail a method based on the solid modelling of each cutter flute [20]. The workpiece surface is discretized in the (XY) plane by parallel lines (called spikes) to the $\mathrm{Z}$ axis (tool axis). The cutter tool model generates a volume which truncates the spikes. The remaining parts of the reference spikes provide a good approximation of the surface finish which can be linked with the cutting parameters. Only a few studies try to link 3D parameters with the machining strategy with the objective of 
optimizing machining parameters [21].

Regarding the integration of the machine tool defaults in surface topography prediction, different research works are proposed. Ehmann et al. [22] introduced the notion of surface shaping system. Using homogeneous transformation from the machine frame to the local cutting edge frame, authors propose a generic model of simulation which includes the tool geometry and the machine tool kinematics. This model is efficient to predict the runout effect on surface topography but no feed rate and force prediction model are proposed. Moreover, no experiment validation is proposed. More recently Li et al. [23] proposed a model of flute movement description in the workpiece coordinate system using homogeneous transformation. Such approach leads to the prediction of the surface topography including cutter revolving precision and static stiffness. However, the real feed rate and the real geometry of the cutting edge are not taken into account.

Few works handle the case of 5-axis milling. Starting from the expression of the trajectory equation of the cutting edge relative to the workpiece and the tool paths, Zhang et al introduced a new and general iterative method to simulate roughness in multi-axis ball-end milling [24] [25]. This method has the advantage of simplicity and is a mesh-independent direct computing method. Effects of the tool inclination and of the cutting mode are in particular investigated. Authors also bring out that surface roughness remains quite unchanged beyond a limit inclination angle. More interesting, the proposed algorithm is used to simulate surface finish of sculptured surfaces, highlighting the effect of the cutting mode on surface roughness. However, the roughness is only apprehended through the $\mathrm{Rz}$ parameter, which does not account for the $3 \mathrm{D}$ effect observed in the simulations.

The prediction of the 3D surface topography in function of the machining conditions remains an important issue in 5-axis machining. Hence, the study presented here aims at formalizing the influence of the cutting conditions on resulting $3 \mathrm{D}$ surface patterns. This is performed thanks to a simulation model based on the well-known N-buffer method. As in sculptured surface machining the feed rates locally vary, the model we proposed is coupled to a velocity prediction model. In order to enhance the effect of both the tool inclination and the cutting parameters, an experimental design is defined taking advantage of the simulation model. The experimental design seeks to characterize factor effects on the resulting $3 \mathrm{D}$ topography through surface parameters. One objective is also to bring out surface parameters that best characterize machined patterns in 5-axis machining.

\section{Prediction of the surface topography}

\section{Simulation model}

The material removal simulation relies on the well-known N-buffer method. This requires the modeling of the surface, the modeling of the tool geometry and the definition of the actual tool trajectory [26]. The workpiece surface is discretized in the (XY) by lines directed along the local normal to the surface. The "hedgehog" so generated is truncated by the cutter tool according to the tool trajectory (figure 
1). The remaining part of the hedgehog defines the 3D topography of the machined surface. The quality of the part modelling is strongly linked with the choice of the sampling parameter. In general, the sampling step chosen is equal to $2 \mu \mathrm{m}$.

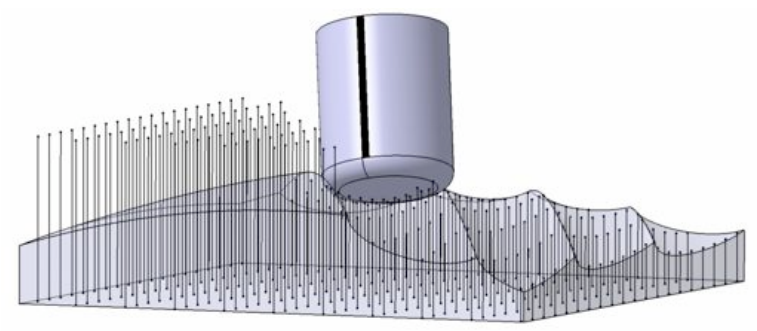

Fig. 1 N-buffer simulation

For its part, the tool is supposed to be rigid, and is approximated by a local meshing (STL format). Only active cutting edges are considered. To ensure a correct approximation of the tool surface, the meshing is performed with a chord error equal to $0.1 \mu \mathrm{m}$ (figure 2 ).

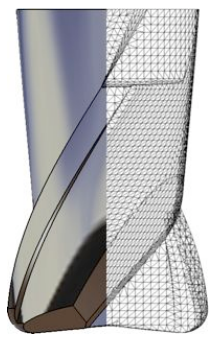

Fig. 2 modeling of the tool geometry

Concerning the tool trajectory, one difficulty is the integration of effects linked to 5-axis machining within a context of high velocities. Indeed, the use of two additional rotational axes leads to two main difficulties during trajectory execution: the computation of the Inverse Kinematical Transformation in real time to define set points corresponding to tool postures, and the synchronization of the rotational axes with the translational ones [27]. Due to kinematical axis capacities, axis velocities vary leading to local feed rate fluctuations which can alter the $3 \mathrm{D}$ pattern. Indeed, the programmed feed rate is seldom reached. In the proposed approach, the simulation of the 3D surface micro-geometry accounts for local variations of the velocity during actual machining. Indeed, the entry of the material simulation model is the actual tool trajectory expressed as a set of tool postures and corresponding feed rates. Actual feed rates can be calculated thanks to a model of velocity prediction developed in a previous work which gives a good estimation of the local feed rate of the tool-teeth [27]. The inputs of the velocity prediction model are the CL file and the characteristics of the machine tool. The CL file gives the set of tool postures defining the trajectory in the part coordinate system (PCS) and the programmed feed rate $\mathrm{Vf}_{\text {prog }}$; tool postures are defined by the coordinates of the tool tip $\left\{\mathrm{X}_{\mathrm{p}}, \mathrm{Y}_{\mathrm{p}}, \mathrm{Z}_{\mathrm{p}}\right\}$ and the axis tool direction components $\{\mathrm{I}, \mathrm{J}, \mathrm{K}\}$. The machine tool characteristics are its architecture, the kinematical axis performances and several CNC parameters (interpolator cycle time, look ahead, rounding parameters...). Given these data, the velocity prediction model generates a set of tool postures with corresponding local feed 
rates $\left\{\mathrm{X}_{\mathrm{p}}{ }^{\mathrm{i}}, \mathrm{Y}_{\mathrm{p}}^{\mathrm{i}}, \mathrm{Z}_{\mathrm{p}}{ }^{\mathrm{i}}, \mathrm{I}^{\mathrm{i}}, \mathrm{J}^{\mathrm{i}}, \mathrm{K}^{\mathrm{i}}, \mathrm{V}_{\mathrm{f}}^{\mathrm{i}}\right\}$ (figure 3 ).

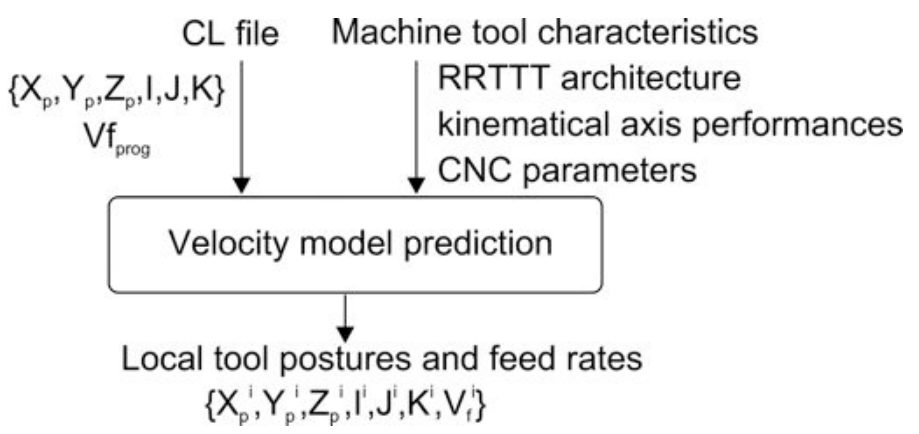

Fig. 3 Prediction of local tool postures and feed rates [27]

Starting from the actual trajectory, the model of material removal simulation thus consists of two steps: calculation of the spindle angular position at each tool posture and trajectory sampling preceding the Nbuffer computation. Given tool postures belonging to the trajectory and corresponding local feed rates, each displacement separating two tool postures is modeled based on the linear interpolation of the feed rate to evaluate its duration. Hence, the time interval separating two postures is calculated as follows:

$$
\Delta t^{i, i+1}=\frac{\sqrt{\left(X_{p}^{i+1}-X_{p}^{i}\right)^{2}+\left(Y_{p}^{i+1}-Y_{p}^{i}\right)^{2}+\left(Z_{p}^{i+1}-Z_{p}^{i}\right)^{2}}}{\frac{V_{f}^{i+1}-V_{f}^{i}}{2}} \text { Eq. (1) }
$$

in each one the local feed rate $V_{f}$ is linearly interpolated (figure 4). If the rotational velocity of the spindle $\Omega$ spindle is supposed equal to the programmed one, the angular positions of the tool axis $\left\{\alpha^{\mathrm{i}}\right\}$ are given by:

$$
\alpha^{\mathrm{i}+1}=\alpha^{\mathrm{i}}+\Omega_{\text {spindle }} \cdot \Delta \mathrm{t}^{\mathrm{i}, \mathrm{i}+1}
$$

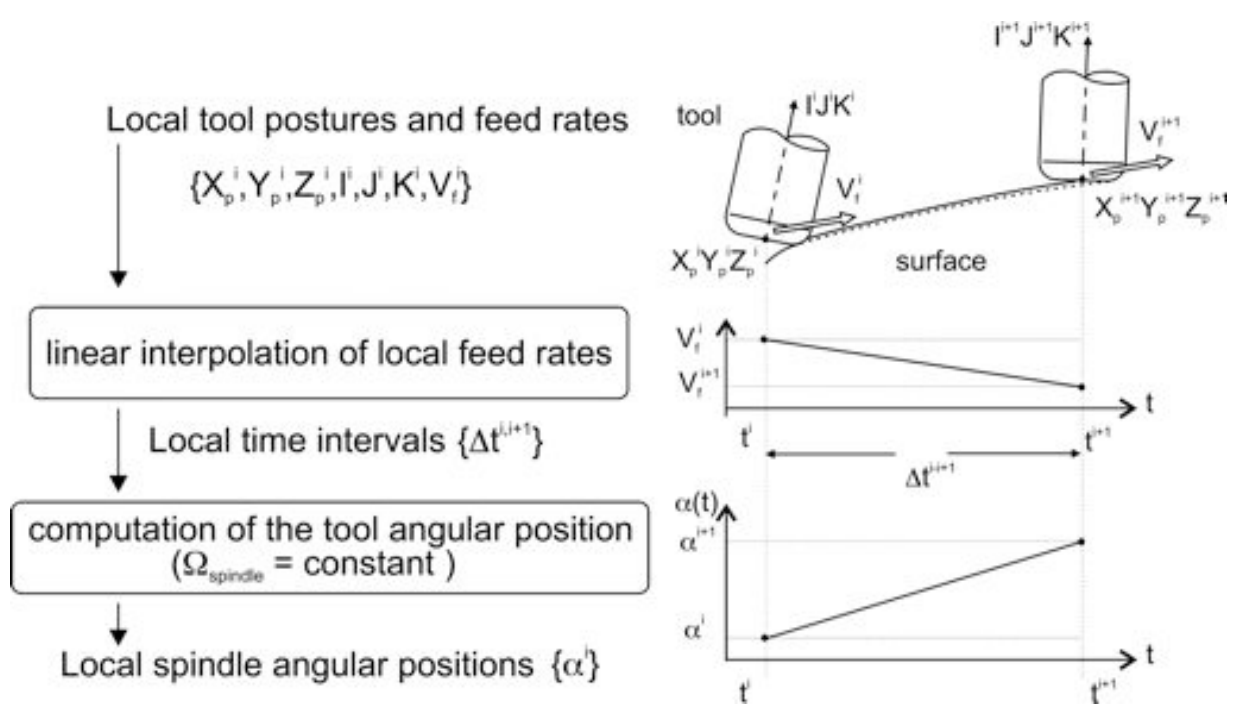

Fig. 4 Computation of spindle angular positions

Once the spindle angular positions are calculated, the trajectory sampling preceding the Nbuffer computation is performed. The elementary trajectory defined between two tool postures is sampled, considering an angular fixed step, d $\alpha$ (figure5). 


$$
\alpha^{*}=\alpha^{i}+N^{*} \cdot d \alpha \quad \text { Eq. (3) }
$$

The value of $d \alpha$ is chosen so that the envelope of the tool movement is entirely represented, and the tool track is not affected by the sampling step. Therefore, the sampling of the position is based on the spindle velocity and on the local predicted feed rates. Based on the angular fixed step, d $\alpha$, the equivalent temporal sampling period, $\mathrm{dt}$, is calculated using the following equation:

$$
d t=\frac{d \alpha}{\Omega_{\text {spindle }}} \quad \text { Eq. (4) }
$$

For each sampling point $\left(\mathrm{N}^{*}\right)$, the local feed rate is thus expressed by:

$$
V_{f}^{*}=V_{f}^{i}+\frac{V_{f}^{i+1}-V_{f}^{i}}{\Delta t^{i, i+1}}\left(N^{*} \cdot d t\right) \text { Eq. (5) }
$$

This yields to the calculation of the sampled tool locations along the elementary trajectory (figure 5):

$$
X_{p}^{*}=X_{p}^{i}+\frac{V_{f}^{*}+V_{f}^{i}}{2}\left(N^{*} . d t\right) \quad \text { Eq. }(6)
$$

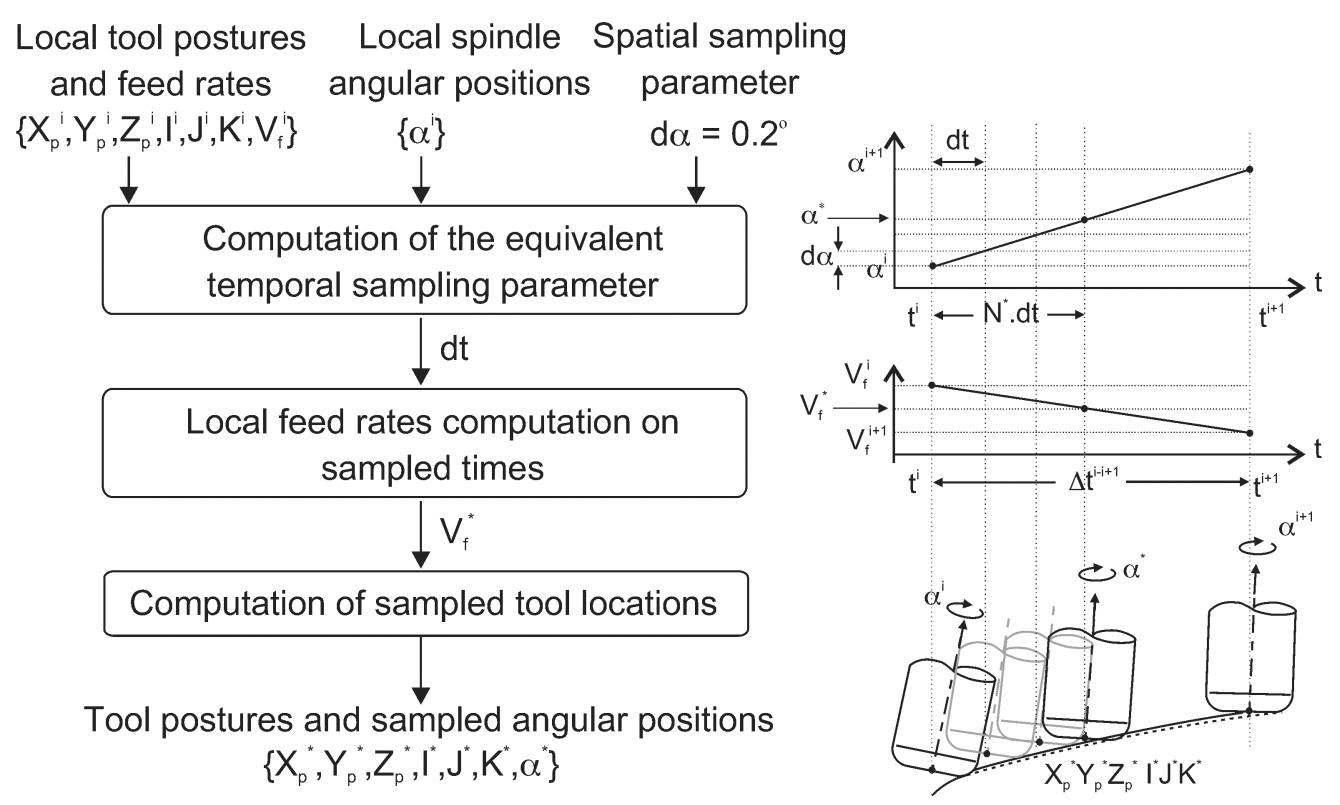

Fig. 5 computation of sampled tool locations

Finally, the simulated machined surface is obtained by computing the intersections between the normal lines of the "hedgehog" and the tool for each configuration $\left\{\mathrm{X}_{\mathrm{p}}^{*}, \mathrm{Y}_{\mathrm{p}}{ }^{*}, \mathrm{Z}_{\mathrm{p}}{ }^{*}, \mathrm{I}^{*}, \mathrm{~J}^{*}, \mathrm{~K}^{*}, \alpha^{*}\right\}$ (figure 5).

A first illustration of the material removal simulation is presented in figure 6 . The colorbar indicates the gap, in millimeters, between the mathematical surface used to compute the tool path and the simulated one after machining. The example concerns the machining of a plane surface using a filleted end milling tool $(\mathrm{R}=5 \mathrm{~mm}, \mathrm{rc}=1.5 \mathrm{~mm})$ considering a maximum scallop height allowed of $\mathrm{hc}=$ 
$0.005 \mathrm{~mm}$. The tool inclination is defined according to figure 7 by the yaw angle $\left(0^{\circ}\right)$ and the tilt angle $\left(1^{\circ}\right)$. To enhance the effect of local variations of the feed per tooth, a linear evolution of the feed rate is set from $4 \mathrm{~m} / \mathrm{min}$, at the beginning of the pass, to $0.5 \mathrm{~m} / \mathrm{min}$ at the end. This influence is clearly visible on the resulting surface topography: the pattern becomes thinner as the feed rate decreases (figure 6). This illustration brings out the major effect of the actual local feed rate in the simulation of machined surface topographies.

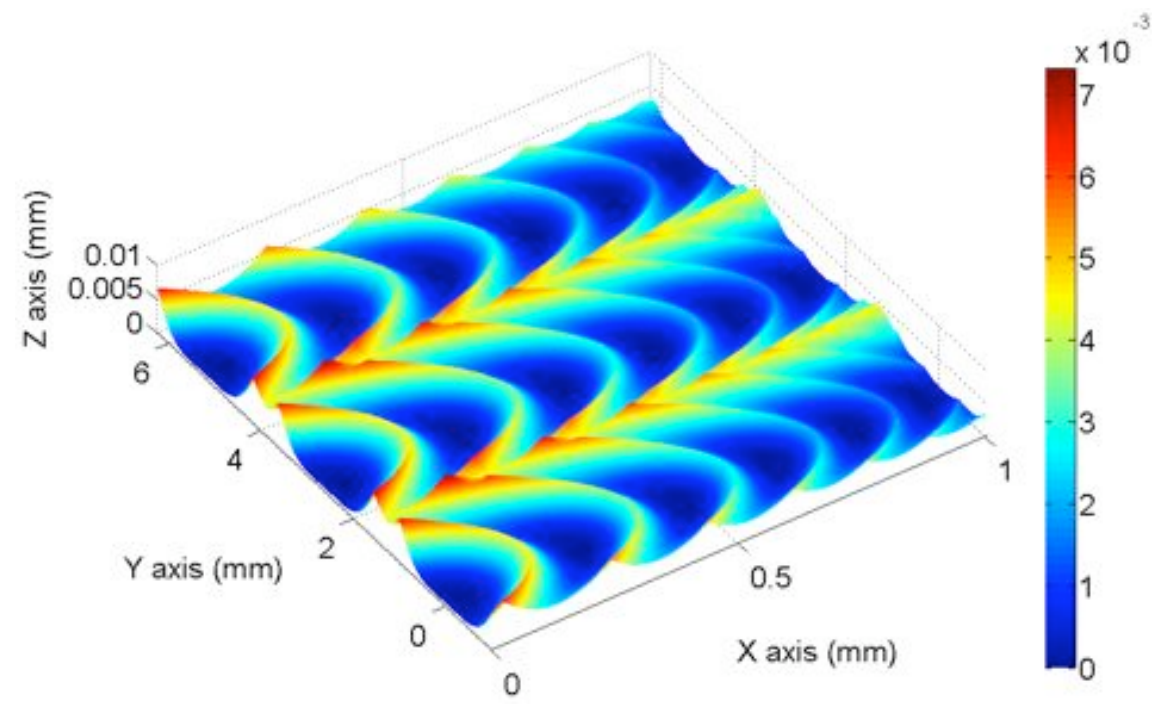

Fig. 6 Topography simulation considering variable local feed rates along a pass

The second illustration is dedicated to the machining of a complex surface in 5axis milling (figure 7) using a filleted end tool $(\mathrm{R}=5 \mathrm{~mm}, \mathrm{rc}=1.5 \mathrm{~mm})$. The surface is defined as an extrusion of a parabola curve $\left(z=-a \cdot x^{2}+d\right.$, with $a=0.004 \mathrm{~mm}^{-1}$, $\mathrm{d}=0.1 \mathrm{~mm}$ ) along the y direction. Cutting conditions are constant: $\mathrm{Vf}=5 \mathrm{~m} / \mathrm{min}$, hc $=0.01 \mathrm{~mm}$, rotational velocity, $\Omega=15000 \mathrm{rpm}$, yaw angle $=0^{\circ}$, and tilt angle $=5^{\circ}$. The machining is performed in a parallel plane mode at $45^{\circ}$ in the $X-Y$ plane. As shown in figure 7 , the resulting pattern is affected by the surface curvature combined with the tool orientation.

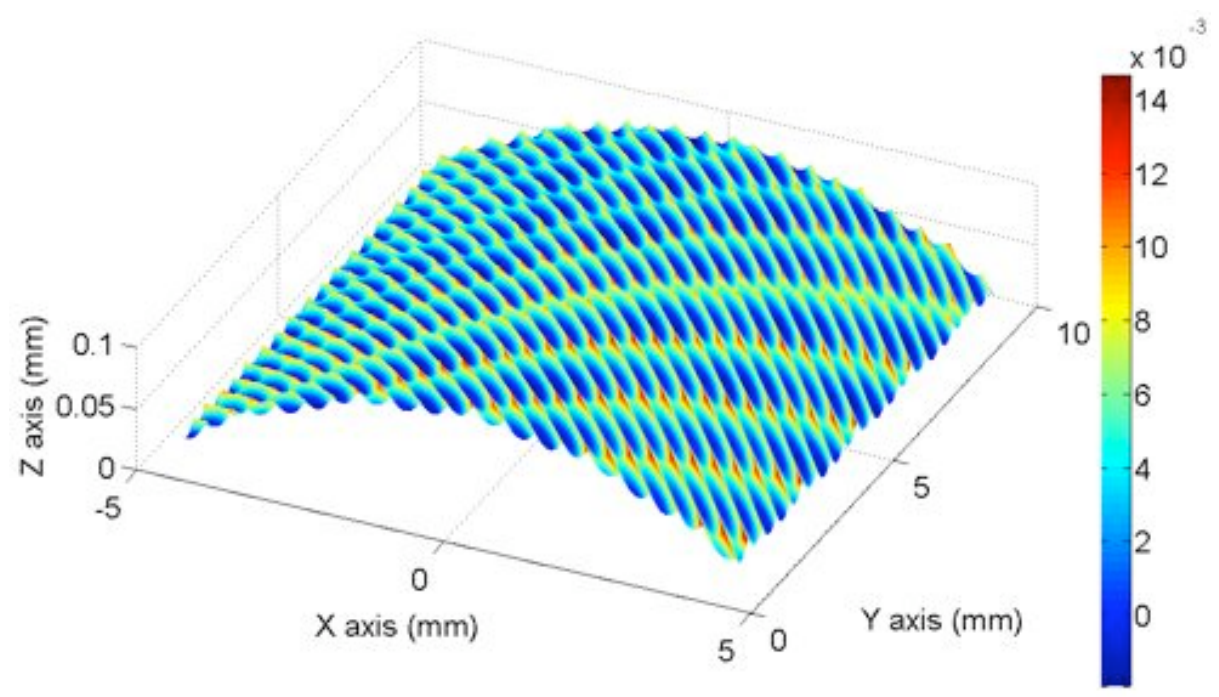

Fig. 7 Topography simulation of a complex surface 


\section{Assessment of the 3D surface topography simulation model}

In order to assess the model, a series of trials is carried out (figure 8), with the aim of comparing various 3D surface patterns obtained by simulations to actual ones.
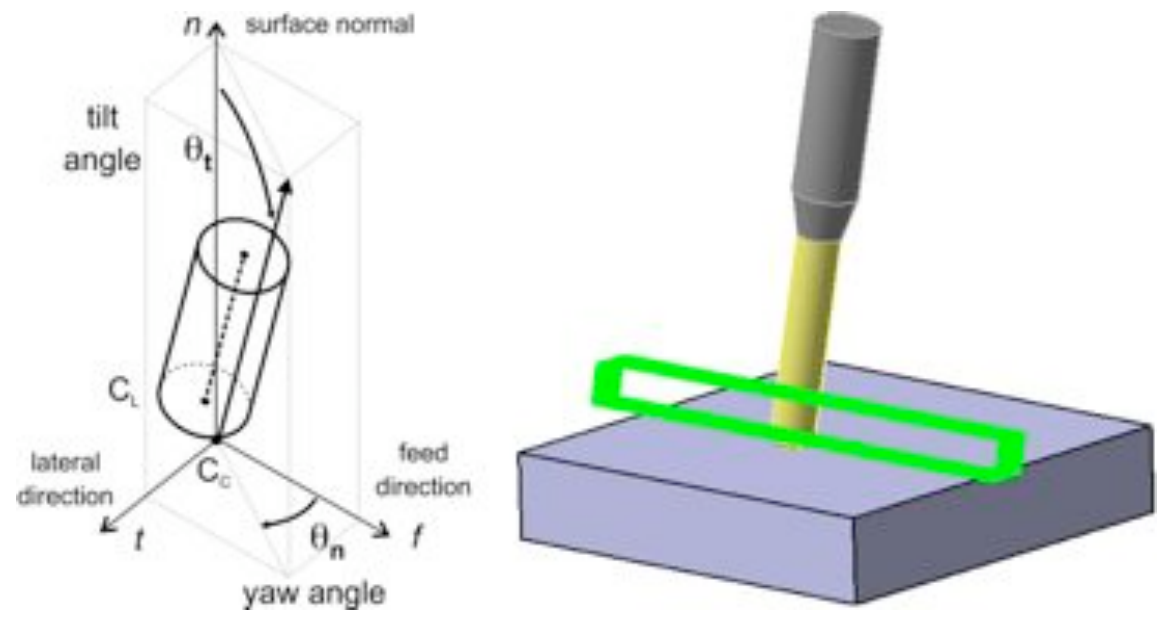

Fig. 8 Description of the trials and tool inclination parameters

For this purpose, a series of plane sweepings of an aluminum alloy with a filletedend mill tool is performed according to variable cutting parameters (table 2). The actual machining is performed on a 5-axis HSM milling centre using a filleted-end milling tool $(\mathrm{R}=5 \mathrm{~mm}, \mathrm{rc}=1.5 \mathrm{~mm})$ with a unique tooth in order to control the geometry of the tooth which contributes to the final imprint.

Tab. 2 Cutting parameters

\begin{tabular}{|c|c|c|c|c|}
\hline Test & Yaw $\left(^{\circ}\right)$ & Tilt $\left(^{\circ}\right)$ & Scallop height $(\mathrm{mm})$ & Feedrate $(\mathrm{m} / \mathrm{min})$ \\
\hline 1 & 0 & 1 & 0,005 & 2 \\
\hline 2 & 0 & 1 & 0,005 & 4 \\
\hline 3 & 0 & 10 & 0,01 & 4 \\
\hline 4 & 20 & 10 & 0,01 & 4 \\
\hline
\end{tabular}

The machined surfaces are measured using an optical instrument (Wyko NT1100 - http://www.veeco.com/). The use of 3D surface parameters is relevant as the topography is clearly anisotropical and tridimensional. To characterize the obtained pattern, the 3D amplitude parameters defined in the standard [ISO 25178] are used. Sz corresponds to the maximum height of the surface and $\mathrm{Sq}$ is the root mean square height of the surface. The functional parameter Std corresponds to the texture direction of the surface. For all cases, 3D patterns and defect amplitudes issued from simulation match the measured ones (figures 9 and 10; table 3). For most cases, deviations are less than 5\% and for a few ones the deviation can reach $9 \%$ due to not optimal cutting conditions, in particular for high values of the yaw and tilt angles.

Tab. 3 Comparison between measured and simulated patterns

\begin{tabular}{|c|c|c|c|c|c|}
\hline Test & Sz $(\mu \mathrm{m})$ & $\mathrm{Sq}(\mu \mathrm{m})$ & $\begin{array}{c}\text { Trans. step } \\
(\mathrm{mm})\end{array}$ & $\begin{array}{c}\text { Long. step } \\
(\mathrm{mm})\end{array}$ & Std $\left(^{\circ}\right)$ \\
\hline
\end{tabular}




\begin{tabular}{|l|c|c|c|c|c|c|c|c|c|c|}
\hline 1 & 4,77 & 4,95 & 1.44 & 1.4 & 2.76 & 2.63 & 0.13 & 0.133 & $2.27^{\circ}$ & $-2.19^{\circ}$ \\
\hline 2 & 8.88 & 9.22 & 1.84 & 1.98 & 2.71 & 2.62 & 0.269 & 0.266 & $-3.92^{\circ}$ & $-3.94^{\circ}$ \\
\hline 3 & 14.9 & 13,5 & 3.06 & 2.97 & 1.21 & 1.18 & 0.267 & 0.266 & $0.02^{\circ}$ & $-0.01^{\circ}$ \\
\hline 4 & 6.15 & 5.6 & 1.2 & 1.25 & 0.46 & 0.43 & 0.264 & 0.266 & $-18.5^{\circ}$ & $-26.4^{\circ}$ \\
\hline
\end{tabular}

However, the longitudinal step as well as the transversal step obtained using the simulated patterns are consistent with those obtained with the measured ones. The longitudinal step is directly linked to the programmed feed per tooth. The transversal step is linked with the programmed scallop height and also with the effective cutting radius.

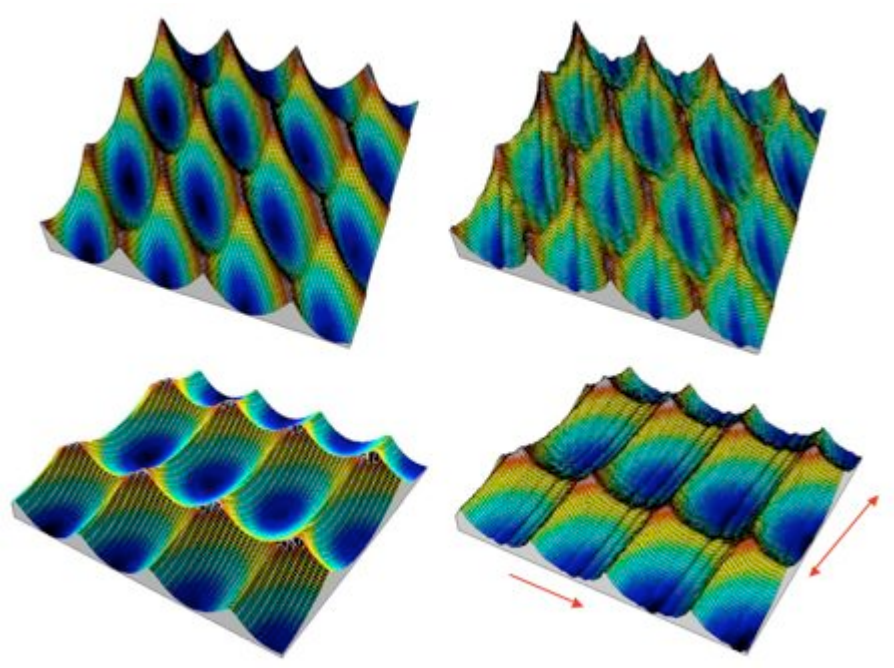

Fig. 9 Simulated (left) and measured (right) 3D patterns (tests 3 and 4)
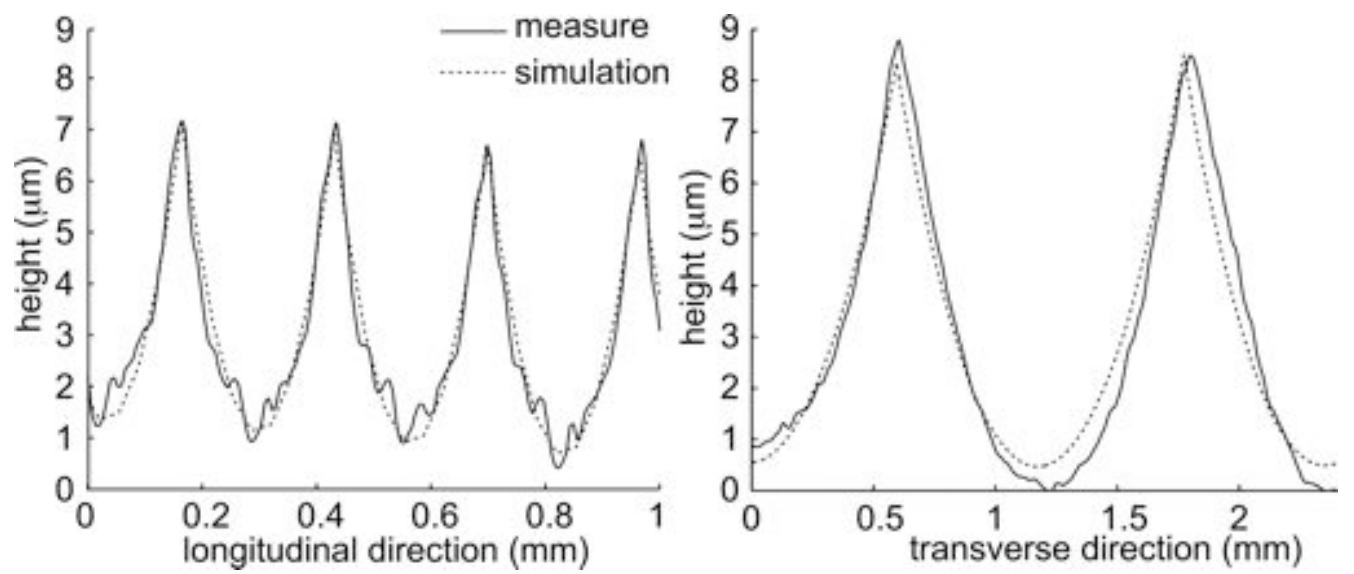

Fig. 10 Simulated and measured profiles (case 3)

Concerning the surface topography, small deviations between simulated and measured data can be observed and may come from the actual tool geometry or/and the cutting process. Despite these comments, the model proposed to predict 3D surface topography is efficient. Hence, the analysis of influent parameters on the surface topography can be conducted through simulations. Furthermore, the use of 3D parameters to characterize surface finish is relevant as they clearly reflect effects observed. 


\section{D surface topography vs machining parameters}

In this section, an experimental design is proposed with the aim of formalizing the influence of the tool axis orientation and the cutting conditions on resulting 3D surface patterns. The objective is also to highlight surface parameters that best characterize machined patterns. The experimental design consists of 4 factors at 2 levels (table 4). The tilt angle and the yaw angle characterize the influence of the tool inclination, whereas the scallop height and the feed rate are the cutting parameters commonly investigated in finish surface studies. The values are representative of classical milling of sculptured surfaces in high-speed machining.

Tab. 4 Parameters of the experimental design

\begin{tabular}{|l|c|}
\hline Factors & Levels \\
\hline Tilt angle $\left(^{\circ}\right)$ & $1-10$ \\
\hline Yaw angle $\left(^{\circ}\right)$ & $0-20$ \\
\hline Scallop height $(\mathrm{mm})$ & $0.005-0.01$ \\
\hline Feed rate $(\mathrm{mm} / \mathrm{min})$ & $2000-4000$ \\
\hline
\end{tabular}

Let $\mathrm{M}_{\mathrm{Fij}}$ be the mean value of the responses of the factor $\mathrm{F}_{\mathrm{i}}$ at the level $\mathrm{j}$, and $\mathrm{M}$ be the mean value of all the responses, the factor effect at the level $\mathrm{j}$ is given by:

$$
E_{\text {Fij }}=M_{i j}-\text { M Eq. (7) }
$$

The evolution of the factor effect at the first level is reported in figure 11 for each surface parameter. As unit and magnitude of the parameters are obviously different, each effect is divided by the mean value.

$$
\mathrm{E}_{\mathrm{Fil}}=\left(\mathrm{M}_{\mathrm{Fi} 1}-\mathrm{M}\right) / \mathrm{M} \quad \text { Eq. (8) }
$$

As the graph distinctly highlights, the yaw angle is the most influent factor on surface topography. This is an interesting result; the tool inclination is definitively the major factor controlling the surface topography. The scallop height is especially significant for amplitude parameters. The feed rate is a secondary parameter, as it actually conditions spatial parameters. Therefore, modifications of feed rate during machining generally affect the 3D surface finish (as shown in figure 6). It is thus essential to integrate local variations of the feed rate in the simulation as proposed in our simulation model. Indeed, when milling sculptured surfaces in 5-axis HSM, the feed rate is seldom the programmed one due to kinematics limits linked to the part geometry (curvature modifications, abrupt variations of tangency...) [27]. Note that some surface parameters are minor as Sku and Sci. Besides, the study of the experimental design shows no correlation between parameters. 


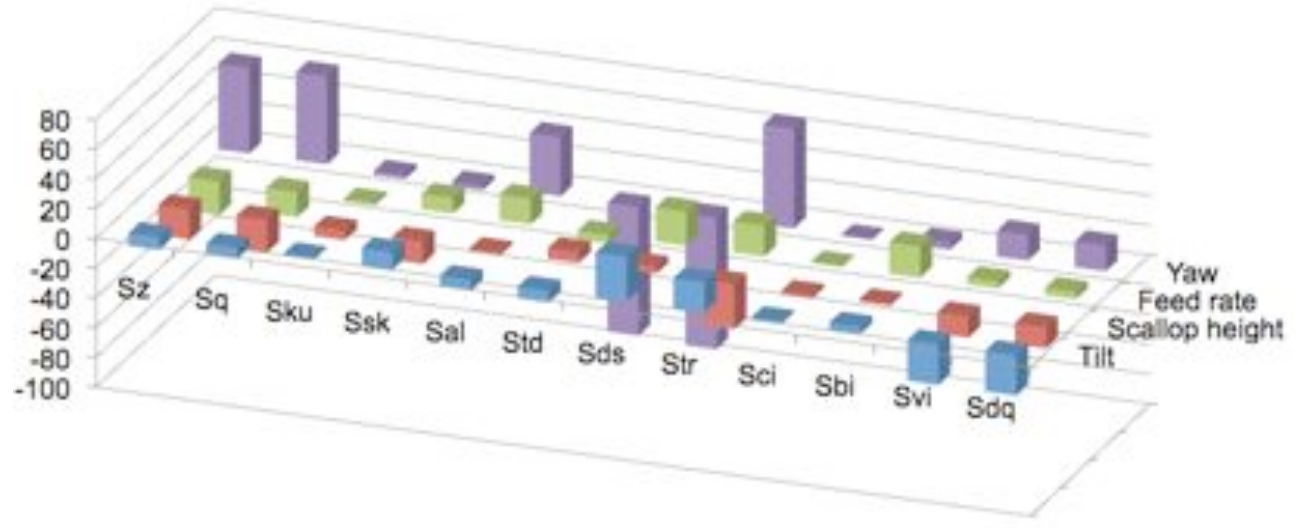

Fig. 11 Factor effects at the first level

The geometry of the pattern, in particular the shape of the valleys is linked to the ratio "corner radius"/"tool radius. This effect is not investigated in this study. Nevertheless, as the influence of the yaw angle is predominant, a specific analysis is developed. A new experimental design is performed considering the same 4 factors as previously. In this design, the levels for the tilt angle, the scallop height and the feed rate remain unchanged, unlike the yaw angle for which the number of levels is modified to $6:\left(0^{\circ}, 2^{\circ}, 5^{\circ}, 10^{\circ}, 15^{\circ}, 20^{\circ}\right)$. The evolution of the effects of the yaw angle is reported in figure 8. Only the surface parameters the most sensitive to the yaw angle effect are considered.

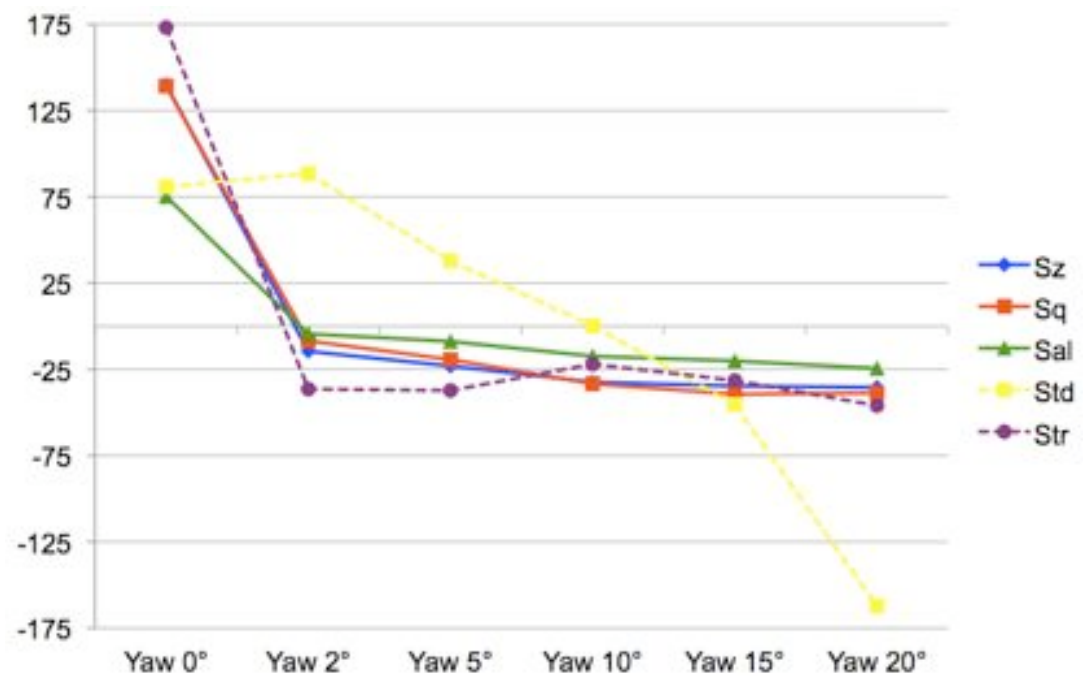

Fig. 12 Effect of the yaw angle

Results enhance the major effect at low values of the yaw angles for most parameters: beyond $2^{\circ}$, the effect is the same. Only the texture direction Std is affected by large yaw angle values. This is not surprising as the texture direction is very sensitive to the slightest change in pattern direction.

Therefore, in 5-axis milling using a tapered-end tool, the resulting pattern is given by the tool geometry and the tool inclination. In particular, the yaw angle is the major factor affecting the topography. Nevertheless, its influence decreases beyond $2^{\circ}$. Hence, to control the amplitude parameters such as $\mathrm{Sz}$ and $\mathrm{Sq}$, it is necessary to first set the yaw angle, then the feed rate and the scallop height next. This enhances the importance of the feed rate prediction all trajectory long, as the 
amplitude parameters of the surface finish are very sensitive to feed rate variations. As the spatial parameters are concerned, Sds and Str are mostly affected by the feed rate and the tilt angle. Functional parameters are in turn not sensitive to the factor in this study. Indeed, functional parameters are mainly controlled by the tool geometry.

\section{Conclusion}

The influence of the machining strategy in 5-axis milling (cutting parameters and tool inclination) on machined surface topography has been investigated in the paper through an experimental design. The surface topography is simulated thanks to a predicting model based on the material removal simulation. The model accounts for variations of the local feed rate which is usual in 5-axis milling of sculptured surfaces using a tapered-end tool. Usually the maximum scallop height allowed is one of the parameters the most used in CAM software to define the 3D surface topography. Results have highlighted that a non null yaw angle provides a pattern for which the notion of cusp only has no more significance. The yaw angle turns out to be the most significant influencing factor. Furthermore, the experiment enhances the influence of the feed rate on amplitude parameters. It is thus important to predict its evolution all trajectory long. We can also conclude on the interest of using surface parameters to describe the surface topography. The step afterwards is the link of such parameters to the workpiece function. Nevertheless, according to the surface parameter retained to characterize the $3 \mathrm{D}$ pattern, the experiment gives information on the influencing machining parameters. The user is thus able to make a choice as regards the expected geometrical surface quality. Further work concerns the integration of the tool run out in the model. The application of the model to the prediction of surface topography on free-form surfaces is also in progress.

\section{Acknowledgements:}

This work was carried out within the context of the working group Manufacturing 21 which gathers 16 French research laboratories. The topics approached are:

- modeling of the manufacturing process,

- virtual machining,

- emerging manufacturing methods.

\section{References}

[1] Dong WP, Sullivan PJ, Stout KJ (1994)

Comprehensive study of parameters for characterizing three dimensional surface topography IV:

Parameter for characterising spatial and hybrid properties. Wear. 178, 45-60

[2] Ganti S, Bhushan B (1995)

Generalized fractal analysis and its applications to engineering surfaces. Wear. 180, 17-34

[3] Thomas TR, Rosen BG, Amini N (1999)

Fractal Characterisation of the anisotropy of rough surfaces. Wear. 232, 41-50 
[4] Roques-Carmes C, Bodin N, Monteil G, Quiniou JF (2001a)

Description of rough surface using conformal equivalent structure concept. Part 1: Stereological Approach. Wear. 248, 82-91

[5] Roques-Carmes C, Bodin N, Monteil G, Quiniou JF (2001b)

Description of rough surface using conformal equivalent structure concept. Part 2: Numerical approach. Wear. 248, 92-99

[6] Saravanakumar P, Manesh KK, Singaperumal M, Ramamoorthy B (2009)

Modelling of fluid continuum considering 3D surface parameters in hydraulic assemblies. Precision Engineering. 33, 99-106

[7] Blunt L, Jiang X, (2003)

Advanced techniques for assessment surface topography: Development of a basis for 3D surface texture standards "surfstand". Kogan Page science, ISBN 1903996112

[8] Novovic D, Dewes RC, Aspinwall DK, Voice W, Bowen P (2004)

The effect of machined topography and integrity on fatigue life. International Journal of Machine Tools and Manufacture. 44,125-134

[10] Ramos AM, Relvas C, Simões A (2003)

The influence of finishing milling strategies on texture roughness and dimensional deviations on the machining of complex surface. Journal of Materials Processing Technology. 136, 209-216

[9] Benardos PG, Vosniakos GC (2003)

Predicting surface roughness in machining: a review. International Journal of Machine Tools and Manufacture. 43, 833-844

[11] Kang MC, Kim KK, Lee DW, Kim JS, Kim NK (2001)

Characteristics of inclined Planes According to the Variations of the cutting direction in highspeed ball-end milling. International Journal of Advanced Manufacturing Technology. 17, 323-329

[12] Baptista R, Antune Simoes JF (2000)

Three and five axes milling of sculptured surfaces. Journal of Materials Processing Technology. $103,398-403$

[13] Axinte DA, Dewes RC, (2002)

Surface integrity of hot work tool steel after high speed milling-experimental data and empirical models. Journal of Materials Processing Technology. 127, 325-335

[14] Ko TJ, Kim HS, Lee SS (2001)

Selection of the machining inclination angle in high-speed ball end milling. International Journal of Advanced Manufacturing Technology. 17, 163-170

[15] Jung JY, Kim CM, Ko TJ, Chung WJ (2004)

Optimization for improvement of surface roughness in high speed machining. Current Advances in Mechanical Design and Production. 8, 953-960

[16] Kim BH, Chu CN (1999)

Texture prediction of milled surfaces using texture superposition method. Computer-Aided Design. 31, 485-494

[17] Bouzakis KD, Aichouh P, Efstahiou K (2003)

Determination of the chip geometry, cutting force and roughness in free form surfaces finishing milling, with ball end tools. International Journal of Machine Tools and Manufacture. 43, 499-514

[18] Toh CK (2004)

Surface topography analysis in high speed finish milling inclined hardened steel. Precision Engineering. 28, 386-398

[19] Chen JS, Huang YK, Chen MS (2005) 
A study of the surface scallop generating mechanism in the ball-end milling process. International Journal of Machine Tools and Manufacture. 45, 1077-1084

[20] Liu N, Loftus M, Whitten A (2005)

Surface finish in high speed, ball nose milling applications. International Journal of Machine Tools and Manufacture. 45, 1152-1161

[21] Quinsat Y, Sabourin L, Lartigue C (2008)

Surface topography in ball en milling process: Description of a 3D surface roughness parameter. Journal of Materials Processing Technology. 195,135-143

[22] Ehmann KF, Hong MS (1994)

A Generalized Model of the Surface Generation Process in Metal Cutting. CIRP Annals Manufacturing Technology. 43, 483-486

[23] Li SJ, Liu RS, Zhang AJ (2002)

Study on an end milling generation surface model and simulation taking into account of the main axle's tolerance. Journal of Materials Processing Technology. 129, 86-90

[24] Gao T, Zhang WH, Qiu K, Wan M. (2006)

Numerical Simulation of Machined Surface Topography and Roughness in Milling Process. Journal of Manufactruing Science and Engineering. 128, 96-103

[25] Zhang WH, Tan G, Wan M, Gao T, Bassir D (2008)

A new algorithm for the numerical simulation of machined surface topography in Multi axis ballend milling. Journal of Manufacturing Science and Engineering. 130, 11003-1/11003-11

[26] Lavernhe S, Quinsat Y, Lartigue C, Meyer R (2008a)

$\mathrm{NC}$-simulation for the prediction of surface finish in 5-axis High-Speed Machining, 3rd CIRP International Conference on High Performance Cutting, Dublin (Ireland), 1, pp 387-396, 12-13 June 2008

[27] Lavernhe S, Tournier C, Lartigue C (2008b)

Kinematical performance prediction in multi-axis machining for process planning optimization. International Journal of Advanced Manufacturing Technology. 37, 534-54

\section{Appendix}

Experimental design

\begin{tabular}{|c|c|c|c|c|}
\hline Test & Yaw angle $\left(^{\circ}\right)$ & Tilt angle $\left.^{\circ}\right)$ & Scallop height $(\mathrm{mm})$ & Feedrate $(\mathrm{m} / \mathrm{min})$ \\
\hline 1 & 0 & 1 & 0,005 & 2 \\
\hline 2 & 0 & 1 & 0,005 & 4 \\
\hline 3 & 0 & 1 & 0,01 & 2 \\
\hline 4 & 0 & 1 & 0,01 & 4 \\
\hline 5 & 0 & 10 & 0,005 & 2 \\
\hline 6 & 0 & 10 & 0,005 & 4 \\
\hline 7 & 0 & 10 & 0,01 & 2 \\
\hline 8 & 0 & 10 & 0,01 & 4 \\
\hline 9 & 20 & 1 & 0,005 & 2 \\
\hline 10 & 20 & 1 & 0,005 & 4 \\
\hline 11 & 20 & 1 & 0,01 & 2 \\
\hline 12 & 20 & 1 & 0,01 & 4 \\
\hline 13 & 20 & 10 & 0,005 & 2 \\
\hline 14 & 20 & 10 & 0,005 & 2 \\
\hline 15 & 20 & 10 & 0,01 & \\
\hline
\end{tabular}




\begin{tabular}{|l|l|l|l|l|}
\hline 16 & 20 & 10 & 0,01 & 4 \\
\hline
\end{tabular}

